\title{
Impacto en la calidad de vida con manejo biorregulador en un paciente con artritis reumatoide.
}

\author{
Martha Maria Giraldo, ${ }^{1}$ Daniela Giraldo Lopez, ${ }^{2}$ Alejandra Guerra, ${ }^{3}$ Lourdes Guerra, ${ }^{4}$ Maria \\ Victoria Llanos, ${ }^{5}$ Marcela Zuluaga ${ }^{6}$ \\ Corresponding Author: Martha Maria Giraldo, mamgiraldo@unal.edu.co \\ ${ }^{1}$ Scomeddih | mamgiraldo@unal.edu.co \\ ${ }^{2}$ Scomeddih | daniii.giraldo@hotmail.com \\ 3 aleguerra2420@gmail.com \\ 4 lougueca@gmail.com \\ 5 vickyllanos8@gmail.com \\ 6.marazull@chdcardio.com
}

\begin{abstract}
Estudio de tipo observacional transversal donde se evalúa la respuesta clínica del paciente con artritis reumatoidea (AR) al manejo con medicamentos inmunomoduladores, multicomponentes: Traumeel, Zeel T, Lymphomyosot, Nuxeel Homaccord, Berbeel Homaccord, Discus compositum, Ubichinon compositum y Gelsemium Homaccord, mediante combinación de diferentes técnicas como la homeosiniatria, acupuntura y la vía oral, implementando cambios en el estilo de vida, por un período de 2 meses. Observándose mejoría en los signos inflamatorios y calidad de vida del paciente. Las mediciones de las variables se hicieron a través de la aplicación las escalas: EuroQol-5D y Escala cuantitativa numérica (ECN). La calificación del dolor describió una disminución de 10/10 en ECN a 4/10 en ECN. Según el termómetro EuroQol-5D la autovaloración de su estado de salud pasó de 20/100 a 80/100. Además, se logró reducir los requerimientos de medicamentos alopáticos.
\end{abstract}

Keywords: Artritis Reumatoide, Medicina integrativa, Caso clínico, Calidad de vida

\section{Introduction}

Paciente de 45 años, con diagnóstico de AR seropositiva desde el 2006, en manejo farmacológico alopático, a pesar del cual presenta aumento de los síntomas de dolor articular en manos, hombros, rodillas, región cervical y lumbar; con pérdida de la autonomía y detrimento de la calidad de vida, lo que lo motiva a consultar en enero de 2021. Como antecedente tiene una cirugía de disco lumbar L2-L3 en 2005 por hernia discal. 
La AR es una enfermedad inflamatoria crónica, de origen desconocido, que se caracteriza por ser una poliartritis simétrica y periférica. Constituye la modalidad más frecuente entre las artritis inflamatorias crónicas y suele ocasionar daño articular y discapacidad física. Se trata de un trastorno de orden general (sistémico) y por ello la AR puede acompañarse de diversas manifestaciones extraarticulares como fatiga, nódulos subcutáneos, afectación pulmonar, pericarditis, neuropatía periférica, vasculitis y anomalías hematológicas. (1).

La clasificación se realizó empleando los criterios de diagnóstico clínicos para AR, concertado en el 2010 por la EULAR y el ACR20 (2). (Imagen 1).

La incidencia aumenta entre los 25 y 55 años. La AR es la más común de las artropatías inflamatorias, afecta al 1\% de la población adulta. La prevalencia más alta se ha encontrado en poblaciones indígenas de los Estados Unidos y la prevalencia más baja corresponde a países orientales. En Latinoamérica se ha reportado una prevalencia cercana al 0.5\% (3). En Colombia, para el 2019, se encontraron 81.386 casos de AR confirmada en paciente de 18 o más años; la prevalencia nacional fue de 0.23 por 100 habitantes, con una incidencia ajustada por la edad de 13.98 (IC 95\% 13.58-14.38) por 100.000 habitantes, de los cuales el $80.4 \%$ son mujeres, con una relación de 5.22 mujeres por cada hombre que la padece; el $40.09 \%$ de los casos corresponden a las edades comprendidas entre los 50 y 64 años. En los hombres, la mayor frecuencia estuvo entre los 60 y 64 años, mientras que en las mujeres se encuentra entre los 55 y 59 años. (4)

Este caso clínico se relaciona con la intervención terapéutica de medicina integrativa que recibió el paciente para el control de los síntomas, observándose ganancia funcional y por ende mejoría del estado emocional, con reintegro a sus actividades cotidianas. Esto nos permitiría establecer un manejo más rápido y eficaz, con menos efectos adversos con relación al uso de los esteroides e inmunosupresores, y un mejor control de la enfermedad. Además, tiene aplicabilidad en la población infantil que presenta este tipo de artropatías inflamatorias.

Adicionalmente tiene un impacto económico en los sistemas de salud, disminuyendo los costos de los nuevos tratamientos, como son el uso de biológicos, y los fármacos convencionales.

\section{Timeline}

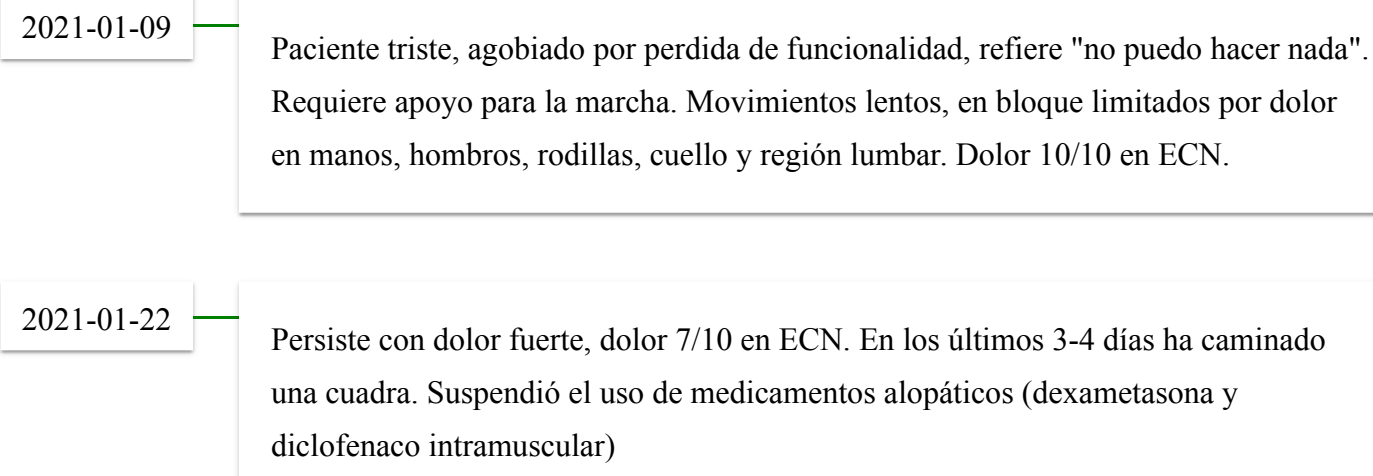



2021-01-29 - Se siente mejor, con ánimo. El dolor bajo, 5/10 en ECN, ya reinició las caminatas que le suben moderadamente el dolor de caderas y rodillas. Persiste leve rigidez en cuello, brazos y manos casa como lavar la loza y cocinar. Dolor 4/10 en ECN. Dolor matutino de los hombros y las rodillas, con disminución de la fuerza en la mano izquierda. biorreguladores hace 1 mes. Ganancia de la movilidad en manos y hombros, dolor 3/10 ECN. Región lumbar y rodillas con dolor 4/10 en ECN. Sin limitación para las actividades.

\section{$\underline{\text { Narrative }}$}

\section{Primera consulta 09/01/2021:}

Paciente masculino de 59 años, mestizo, se desempeña en oficios varios, acude a consulta por primera vez el 09 de enero de 2021, por crisis de dolor articular generalizado de 20 días de evolución, principalmente en manos, hombros, rodillas, cuello y región lumbar, que le generan severa limitación funcional, y es agravado con la movilidad.

El paciente califica el dolor 10/10 en escala cuantificada numérica (EN o ECN) (5). Se automedica con dexametasona intramuscular 1 vez por semana durante 3 semanas y diclofenaco intramuscular de una a dos veces al día, según dolor.

\section{$\underline{\text { Antecedentes patológicos (imagen 2) }}$.}

- AR diagnosticado en 2006

- Hipertensión arterial diagnosticada en 2015

Antecedentes traumáticos:

- Trauma a nivel de glúteo por inyección (en 1971)

Antecedentes quirúrgicos:

- Cirugía de columna por hernia discal en 2005

Antecedentes ambientales y_psicosociales: 
- Escolaridad: Secundaria incompleta

- Casado, dos hijos (hombre y mujer).

- Ocupación: Oficios de construcción obra blanca.

- Residencia: Área urbana.

- Tóxicos: Niega

- Actividad física: Sedentario

- Dieta: Prefiere alimentos dulces, harina de trigo con predominio de pan, lácteos, consumo de aceites refinados. Pocas frutas, no consume verduras.

\section{$\underline{\text { Antecedentes farmacológicos: }}$}

- Prednisolona $10 \mathrm{mg}$ /día, ácido fólico $1 \mathrm{mg} /$ día, sulfasalazina 500mg/día, losartan $50 \mathrm{mg}$ cada día.

\section{Antecedentes familiares:}

- Familia compuesta por padres (fallecidos) y 6 hijos.

Cinco mujeres y un hombre, siendo el paciente el cuarto hijo.

Las hermanas \#1 y \#6 tienen AR.

La hermana \#1 inició cuadro de AR deformante después del parto que le causó postración, ya fallecida; y la hermana \#6 inicio con AR después de donación de sangre para la hermana \#1.

Cuatro hermanas son hipertensas.

\section{Revisión por sistemas:}

Orinas concentradas, deposiciones secas una vez al día, patrón de sueño superficial e intermitente por dolor.

Examen físico primera consulta 09 de enero 2021:

Paciente con afecto triste, agobiado, por sentir que no puede mover su cuerpo "no puedo hacer nada". Acompañado de su hermana, quien lo ayuda en apoyo para la marcha, retirar prendas de vestir y subir a la camilla. Los movimientos son lentos, en bloque y limitados por dolor. Dolor 10/10 en ECN 
Presión arterial:130/80 mmHg

Frecuencia cardiaca: 92 latidos por minuto

Frecuencia respiratoria: 18 respiraciones por minuto

Temperatura: $36.1^{\circ} \mathrm{C}$

Peso: $69 \mathrm{~kg}$

Talla: 1.60 metros

Índice de masa corporal: 26.9 Sobrepeso.

Rostro con piel pálida y seca. Mejillas enrojecidas. Boca con lengua roja saburra, de tamaño adecuado, y algodonosa. Pulso lleno y resbaladizo. Manos temblorosas, articulaciones interfalángicas engrosadas y rígidas, limitadas para la movilidad. Atrofia de la eminencia tenar. Congestión de 4to y 5to pulpejos bilaterales. Aumento de diámetro de interfalángicas distales de cuarto y quinto dedo bilateral. Desviación ventral de falange distal 5to dedo bilateral. Se observa cambios en la coloración con eritema distal y palidez en falange media.

Percepción subjetiva de su temperatura: Refiere calor

Se realizó evaluación con escala EuroQol-5D (6), aplicada en dos consultas diferentes. (Imagen 3 y. 4).

Manejo instaurado en la primera consulta:

Biopuntura en puntos de tonificación a nivel de V11, V15, V17, V23 y V52; y en puntos de dolor (Ashi) en columna con Ubichinon Compositum, Discus Compositum, Traumeel.

Detoxificación oral con Lymphomyosot, Berbeel Homaccord, Nuxeel Homaccord.

30 gotas de cada uno en una botella de 1000cc agua por día por 2 meses.

Manejo de dolor ambulatorio:

Zeel-T tableta sublingual 3 veces al día, el primer mes.

Traumeel tableta sublingual 3 veces al día, el primer mes. 
Cambios alimentarios: Evitar alimentos que aumentan la inflamación, lácteos, trigo, avena, maní, soya y cerdo.

\section{Parte médico 22 de enero 2021}

Evolución: Persiste con dolor fuerte, dolor 7/10 en ECN. En los últimos 3-4 días ha caminado una cuadra. Suspendió el uso de medicamentos alopáticos (dexametasona y diclofenaco intramuscular)

Tratamiento:

Biopuntura

- Gelsemium Homaccord en cuello. V11 y V15

- Traumeel + Discus compositum en área lumbar. V20, V23, V52.

- Zeel T + Traumeel en rodilla. E36 y E34.

\section{Parte médico 29 de enero 2021}

Evolución: Se siente mejor, con ánimo. El dolor bajo, 5/10 en ECN, ya reinició las caminatas que le suben moderadamente el dolor de caderas y rodillas. Persiste leve rigidez en cuello, brazos y manos.

Tratamiento:

Biopuntura

- Zeel-T + Traumeel: E36, E34, V23, V52

- Gelsemium Homaccord: V11 y V17

Parte médico 19 de febrero 2021

Evolución: Refiere sentirse mucho mejor en la movilidad de las manos, realizando labores en casa como lavar la loza y cocinar. Dolor 4/10 en ECN. Dolor matutino de los hombros y las rodillas, con disminución de la fuerza en la mano izquierda.

Tratamiento: 
Biopuntura

- Gelsemium Homaccord: V11, V15, V17 y ID3

- Zeel T + Traumeel: V23, V20, B6, E36 y B1

Parte médico 14 de mayo de 2021

Evolución: Paciente refiere que ha vuelto a comer la dieta común, sin restricción.

Toma a diario prednisolona $10 \mathrm{mg} /$ día + acetaminofén $500 \mathrm{mg}$ dos al día.

Hace más de un mes no ha podido comprar los medicamentos homotoxicológicos.

Mayor ganancia de la movilidad en manos y hombros, con dolor 3/10 en ECN.

En región lumbar y rodillas persiste con dolor 4/10 en ECN. Sin limitación para las actividades caseras.

Tratamiento:

Biopuntura

- Gelsemium Homaccord: V11, V15, V17 y ID3.

- Zeel-T + Traumeel: V 23, V 20, B6, E36, B10.

Nos basamos en la historia clínica aportada por el paciente en su cita inicial. Donde se menciona, por el médico tratante el diagnóstico de AR, desde el 2006. Recibiendo manejo alopático como tratamiento para la AR. Por todo lo anterior se limita el establecer diagnósticos diferenciales.

No se contó con exámenes paraclínicos para el seguimiento y determinar la actividad inflamatoria de su patología. Además, la pandemia impidió la asignación de citas por parte de su EPS, para la realización de estos y control por reumatología. Contando solo con evaluación por Medicina general durante este tiempo.

Nuestros mayores desafíos fueron de tipo farmacológico pues teníamos barreras económicas muy importantes, que impedían el seguimiento más continuo, así como la adquisición de medicamentos.

Los factores para definir mal pronóstico de AR son:

- Factores sociodemográficos como sexo femenino, edad, bajo nivel de estudios y genotipo DR (HLA DR4 o epítopo compartido). 
- Factores dependientes de enfermedad: Factor reumatoideo positivo, número elevado de articulaciones tumefactas, duración de la artritis, actividad inflamatoria elevada (clínica, PCR y VSG), afectación precoz de grandes articulaciones, grados elevados de discapacidad, presencia precoz de erosiones radiológicas y manifestaciones extraarticulares.

- Factores dependientes del tratamiento: Inicio precoz de tratamiento con fármacos modificadores de la enfermedad y tratamiento prolongado con los mismos. (7)

Teniendo en cuenta que no contamos con resultados de paraclínicos, no es posible definir el pronóstico del paciente.

Durante el seguimiento del paciente se tuvo oportunidad de realizar una primera cita y 6 citas de control. Cada consulta y su manejo se orientó de acuerdo a la evolución clínica y la sintomatología en ese momento. En los partes médicos anexados se menciona las cinco consultas más representativas para una mejor comprensión sintetizada de este caso clínico.

El tratamiento se enfocó en detoxificación, drenaje e inmunomodulación. Se reforzó la educación en cambios dietarios, además de medicamentos en puntos de biopuntura principalmente en las zonas de dolor. En la Imagen 5 se exponen en su totalidad todos los medicamentos usados en cada una de las consultas.

Se observó mejoría de su estado clínico caracterizado por:

- La calificación del dolor describió una disminución de 10/10 en ECN a 4/10 en ECN.

- Según el termómetro EuroQoL-5D de autovaloración de su estado de salud pasó de 20/100 a $80 / 100$

- Se observó ganancia en la autonomía al lograr realizar labores en relación con autocuidado, lo que representó una participación positiva y activa en la dinámica familiar.

La tolerancia al uso de los medicamentos se evalúa de forma subjetiva al preguntar al paciente si había presentado alguna incomodidad o síntomas de malestar con el uso de biorreguladores empleados.

No se reportaron efectos adversos y colaterales, ni crisis curativas.

\section{Perspective}


El paciente manifestó sentirse complacido con su mejoría. Expresando: "volví a vivir", "mejoró la movilidad".

\section{$\underline{\text { Discussion }}$}

Paciente llega a la primera consulta con gran limitación funcional principalmente en articulaciones de manos, hombros y rodillas, con limitación para realizar ninguna actividad física; iniciando tratamiento con Traumeel, Discus Compositum y Ubichinon compositum, posteriormente se asoció Gelsemium Homaccord y Zeel T continuando con la aplicación de Traumeel y Discus Compositum en puntos de biopuntura en cada consulta. Se dejó para manejo ambulatorio Zeel T oral en asociación con Traumeel oral tres veces al día y detoxificación oral con Lymphomyosot gotas, Nuxeel Homaccord gotas y Berbel Homaccord gotas.

Traumeel ayudó al paciente a aliviar el dolor articular y a recuperar la movilidad de las mismas, regulando el proceso inflamatorio propio de la artritis reumatoidea, ya que este medicamento biorregulador reúne diversos componentes que tratan la inflamación y promueven la recuperación de tejidos de formas diversas; Traumeel reduce la producción de citoquinas proinflamatorias como IL-1, IL-6, IL-8 y TNF- $\alpha$ las cuales son las que producen un ambiente proinflamatorio en la articulación. Por otro lado, el Traumeel estimula la formación de citoquinas antiinflamatorias como el TGF- $\beta$, el cual es importante para la regeneración del tejido articular. Induce Th3. Además, mediante la síntesis de TGF- $\beta$ se evita que otros linfocitos T proinflamatorios apoyen el proceso inflamatorio de esta enfermedad. De esta manera Traumeel restaura el equilibrio de las citoquinas, logrando así disminuir o en algunos casos eliminar la inflamación. Este medicamento vuelve a jugar un papel importante en la reducción de la inflamación ya que el NF-k $\beta$ se activa en respuesta a una amplia gama de estímulos dentro de ellos están incluidas las citoquinas proinflamatorias, en particular TNF- $\alpha$ e IL-1; por lo tanto, inhibe indirectamente la activación de NF-k $\beta$ por sus efectos sobre las citoquinas proinflamatorias.

Dentro de los componentes de Traumeel están el Árnica, la Caléndula, el Aconitum, la Chamomilla y el Hamamelis Hypericum los cuales tiene efectos analgésicos y nos ayudan a modular los dolores articulares en forma rápida. (8)

La hipoxia se ha involucrado también en la patogénesis de la artritis reumatoide, esta se da como resultado de una angiogénesis desregulada, una función mitocondrial alterada y la misma inflamación, lo que lleva a una crisis bioenergética. La hipoxia genera reducción en la respiración mitocondrial, producción excesiva de especies reactivas de oxígeno (ROS) y acumulación de mutaciones en el ADN mitocondrial generando así disfunción mitocondrial y creando un círculo vicioso que solo genera mayor inflamación. (9). Es preciso entonces que al ser la mitocondria la encargada de la regulación de la bioenergética y el metabolismo celular se haya utilizado el

Ubichinon Compositum. El cual modula la respuesta inflamatoria ya que regula la función de la cadena respiratoria mitocondrial, estimula la cadena de citocromo oxidasa de la mitocondria para la producción ATP y disminuye la producción de especies reactivas del oxígeno (10). La disfunción mitocondrial a nivel de los sinoviocitos en la artritis reumatoidea amplifica la capacidad de 
respuesta a la inflamación de los condrocitos inducida por citoquinas mediante la producción de ROS.

El Discus Compositum está compuesto por cofactores vitamínicos, minerales, plantas y factores metabólicos biorreguladores como protector activo de la matriz extracelular osteocartilaginosa. (11)

El Discus Compositum cumple funciones de inmunomodulación activando la respuesta inmune. También detoxifica la matriz extracelular mejorando el terreno acidófilo, la capacidad antioxidante y la respiración celular, lo que activa la producción de energía. Como soporte orgánico mejora la función respiratoria reforzando las fibras de colágeno. A nivel de sintomatología clínica reduce el dolor neurálgico y el espasmo que puede causarse por degeneración del disco intervertebral, que puede progresar a prolapso, extrusión y secuestro del disco, que en ocasiones puede terminar en compresión de una raíz nerviosa con la consecuente neuropatía. (11)

El Discus Compositum también activa el proceso metabólico del disco intervertebral, protegiendo y recuperando la flexibilidad de este, desencadenada por múltiples causas como: degeneración discal y vertebral, malas posturas, predisposición genética, debilidad muscular y pérdida estructural. (11)

El Discus compositum reduce de manera eficaz los dolores asociados a los procesos degenerativos de la columna, regulando los procesos inflamatorios del disco intervertebral. Favorece la absorción de nutrientes mejorando la irrigación y la microcirculación. Protege la matriz de procesos inflamatorios al favorecer la síntesis de colágeno, disminuyendo la destrucción del tejido. (11).

Todas estas acciones se vieron reflejadas en nuestro paciente como él lo manifestó en sus diferentes citas y al observarse mejoría de su principal síntoma como fue el dolor, pasando de un nivel inicial de 10/10 a 3/10 en ECN a final del tratamiento, corroborando los hallazgos mencionados en los estudios realizados anteriormente (12), y también observándose mejoría de la rigidez de sus articulaciones. Este medicamento además ayudó tanto en su proceso de hernia discal, patología que menciona el paciente como antecedente, y para el tratamiento de su enfermedad actual, la AR.

Se utiliza en el manejo del caso clínico Zeel $\mathbf{T}$ inyectable, medicamento que se usa generalmente en la osteoartritis y como condroprotector.

El Zeel T, por la amplia utilización demostrada de su papel reparador del cartílago articular y de favorecer la producción de condroitín sulfato, queratán sulfato y ácido hialurónico para reparar el tejido articular y modular la producción de COX 2 en la inflamación. Útil en casos de artrosis deformante, dolores neurálgicos y reumáticos de las articulaciones (especialmente agravadas por el frío y la humedad) (10).

Los efectos antiinflamatorios del Zeel T están dados por la inhibición de las dos principales vías oxidativas enzimáticas del ácido araquidónico, que es generado en los procesos inflamatorios, estas vías son: 5-lipooxigenasa (LOX) y la vía de la ciclooxigenasa (COX), lo que afecta los niveles de leucotrienos, lipoxinas, prostaglandinas y tromboxanos, produciendo una disminución en el dolor 
artrítico y de la misma inflamación. Además del efecto antiinflamatorio, el Zeel T tiene efecto en la inmunidad. Estimula la producción de TGF- $\beta$ que es regenerador y modula proceso inflamatorio, modula metaloproteinasas, evita angiogénesis del cartílago e incluso se podría pensar que regula las vías de la sinovia. El TGF- $\beta$ incrementa la formación de fibras colágenas (13).

El Gelsemium Homaccord pertenece a la red miofascial de miembro superior y hombro, además sus propiedades ansiolíticas, antiinflamatorias, analgésicas e inmunorreguladoras favorecen al cuadro clínico del paciente. (14).

El gelsemium sempervirens tiene capacidad antioxidante ya que posee un efecto citoprotector al disminuir las ROS y activa caspasas (15).

Para este paciente, se consideró ideal realizar detoxificación y drenaje para iniciar un proceso biorregulador en un terreno biológico adecuado, en este caso se usó Lymphomyosot como favorecedor de la detoxificación y drenaje de la matriz extracelular (MEC), además sus propiedades reducen la acidificación de la MEC y favorecen los procesos de reparación tisular, también modula la actividad de metaloproteinasas involucradas en el proceso inflamatorio de la MEC. (10). Es importante mencionar que el Lymphomyosot se ha visto involucrado en la modulación del sistema inmune, ya que modula la activación del NF- $\mathrm{k} \beta$ el cual está involucrado en la regulación de la transcripción de genes que codifican citoquinas proinflamatorias. Otros efectos a nivel del sistema inmunológico podrían ser asociados a la modulación del IFN- $\gamma$, IL-10 y IL-8 (citoquinas proinflamatorias). Otro efecto benéfico para este paciente tanto en la mejoría del dolor y la respuesta inflamatoria sería dado por su participación en la modulación de enzimas proinflamatorias como las pertenecientes la vía de la ciclooxigenasa 1 y 2, modula la síntesis de prostaglandinas E2 e histamina, además tiene efectos sobre la síntesis y liberación de óxido nítrico. (16)

Cabe mencionar algunos de sus componentes involucrados en el Lymphomyosot y que cumplen con estas funciones, como es el caso de la fummaria officionales que estimula el sistema emuntorial, es antioxidante y tiene efectos antiinflamatorios al modular la vía de la ciclooxigenasa (17).

Otro de sus componentes es la Veronica officinale la cual disminuye la actividad de transcripción de NF-k $\beta$ (18). El Nastutium officinale tiene efectos antioxidantes a nivel hepático, mejora la actividad de la catalasa y superóxido dismutasa en los tejidos del hígado, tiene actividad antiinflamatoria al disminuir el edema y daño tisular, protegen contra el aumento de óxido nítrico y TNF- $\alpha$. (19). La Smilax tiene efectos antiinflamatorios, inmunomoduladores, antioxidantes, hepatoprotectores, protección cardiovascular y antiviral. (20).

En cuanto al Berbeel Homaccord, el Berberis vulgaris con su principal componente: la Berberina, juega un papel importante en el efecto antiinflamatorio e inmunomodulador a través del cambio de la respuesta inmune celular de los Th2, actuando en la inducción de linfocitos T reguladores, con inhibición de citoquinas proinflamatorias (IL-1, TNF e IFN- $\gamma$-IL 6) y la estimulación de IL-4 e IL$10(21)$. 
Otra acción antiinflamatoria del berberis vulgaris se da por inhibición de la DNA sintetasa en la actividad de los linfocitos. Tiene actividad anti-complemento, al inhibir in vitro la proliferación de linfocitos en sangre periférica al estimular la Fito hemaglutinina concavalina (22). También interviene induciendo la apoptosis en células presentadoras de antígenos y en otras células efectoras. Todas estas funciones ya mencionadas del berberis nos ayudan a controlar la respuesta inflamatoria alterada en los pacientes que cursan con AR.

Se han encontrado nuevas funciones del berberis sobre la actividad antimicrobiana, anti cáncer y otros efectos inmunomoduladores. Así como efectos en la hipercolesterolemia y actúa sobre los canales de calcio intracelular que intervienen sobre el sistema cardiovascular, pero aún se encuentra en estudio, con efecto antihipertensivo y relajante del músculo cardiaco, actuando sobre los receptores agonistas $\alpha-1$ aplicables a la prevención de insuficiencia cardiaca, hipertrofia cardiaca y arritmia. Lo anterior beneficiaría a nuestro paciente en el manejo de su hipertensión arterial, disminuyendo el desarrollo de manifestaciones extraarticulares importantes sobre el sistema cardiovascular en la AR, además tiene acciones sobre el desarrollo del síndrome metabólico. (21).

También se usó en el proceso de detoxificación el Nuxeel Homaccord tiene un mecanismo de acción la detoxificación hepática y gastrointestinal, controla la inflamación de la matriz extracelular de las serosas y mucosas del sistema gastrointestinal, hepático y de vías biliares, tiene psicotrópica controlando los síntomas emocionales y mentales dados por la sobrecarga y acumulación de homotoxinas. (10)

Una de las fortalezas de este caso es haber podido documentar la intervención médica y su evolución usando la medicina biorreguladora, posterior al uso de medicina alopática. El enfoque basado en la historia del paciente permitió orientar hacia el manejo de la inflamación crónica persistente, asociado a una alteración de la función mitocondrial post lesión y manejo quirúrgico de la patología lumbar.

Se cuenta con mediciones cuantificables de la calidad de vida del paciente que permiten ser objetivos frente a la evolución clínica. La aplicación de escalas de medición (EuroQol-5D y Escala de cuantificación numérica del dolor) nos permitirá comparar y estandarizar otros trabajos relacionados con esta patología y evaluar los resultados con la intervención de medicina integrativa.

La disponibilidad de diferentes presentaciones de los medicamentos nos permitió hacer uso de las herramientas terapéuticas como la vía oral y la biopuntura.

Una limitación importante de nuestro caso fue que no contamos con paraclínicos o ayudas diagnósticas que nos permitieran evaluar o verificar con parámetros bioquímicos e inmunológicos la mejoría de la respuesta inflamatoria en el paciente. Lo que nos llevó a hacer una evaluación objetiva basada en la evolución y el seguimiento clínico evidenciado por la mejoría de los cambios inflamatorios a nivel de las articulaciones afectadas, y mejoría en la funcionalidad y movilidad del paciente.

\section{Conclusion}


Dentro de la metodología para abordar este paciente, con una visión integradora de diferentes conocimientos, experiencias, habilidades, aptitudes, se resalta la importancia de centrarnos en el paciente, en su historia de vida que nos permitió conocer cómo evolucionó, en su caso particular, cada evento y así mismo se realizó un análisis para definir qué puntos y con cual terapéutica se debería enfocar; la causa de su consulta fue una oportunidad para intervenir con medicina holística y a su vez nos permite medir la respuesta al tratamiento.

Se observa que un paciente intervenido con la medicina biorreguladora genera un impacto positivo en su calidad de vida. Resaltando que se observa mejoría clínica importante con el tratamiento recibido durante 2 meses: 7 sesiones de biopuntura semanales y uso de medicamentos biorreguladores vía oral.

En este caso, no se observaron efectos secundarios, lo que generó mayor adherencia, confianza en el médico y en el producto que se usó.

Nos permitió realizar un manejo integrativo del paciente utilizando medicamentos biorreguladores basados en medicamentos homeopáticos a diferentes diluciones y complementarlos con los principios de la Medicina Tradicional China.

Al usar herramientas de medición estandarizadas nos permitiría realizar estudios comparativos y aplicarla a poblaciones mayores, evaluando la respuesta terapéutica y clínica al uso de los diferentes medicamentos biorreguladores de una manera más significativa estadísticamente.

La inflamación crónica de la artritis reumatoide está dada por una interrelación compleja de los factores genéticos, ambientales, inmunitarios, además una posible asociación a alteraciones en la microbiota y una transgresión de la auto tolerancia.

En la fase preclínica asintomática de la enfermedad de artritis reumatoide, el sistema inmunológico se caracteriza por una reducción de la auto tolerancia y producción de autoanticuerpos. En la fase clínica las células inmunitarias innatas y adaptativas se infiltran en las articulaciones sinoviales y producen síntomas de dolor y rigidez; la membrana sinovial es infiltrada por linfocitos $\mathrm{T}$, linfocitos B y macrófagos. Al progresar la artritis reumatoide, las células inmunes y los fibroblastos sinoviales producen un ambiente proinflamatorio en la articulación, que conduce a la destrucción articular. Las citocinas específicas que participan son TNF- $\alpha$, IL-1 e IL-6 de macrófagos, IL-7 e IL-15 de las células T de memoria, IL-18, factor estimulante de colonias de granulocitos y monocitos (GM-CSF) y TGF- $\beta$ de fibroblastos sinoviales. Este complejo medio de citoquinas atrae más células inmunes, lo que promueve angiogénesis anormal, osteoclastogénesis, alteración vascular, lo que conduce a los efectos sistémicos. (23). 
Finalmente, el fallo mitocondrial de la artritis hace que el proceso de reparación del ADN mitocondrial sea insuficiente y la célula se ve obligada a funcionar con mitocondrias dañadas. Además, las especies reactivas de oxígeno estimulan la secreción de metaloproteinasas por parte de los sinoviocitos similares a fibroblastos, inhiben la síntesis de proteoglicanos del cartílago y aceleran la reabsorción ósea.

En el caso de este paciente, por las características de su enfermedad y todos los factores epigenéticos asociados se favorece la detoxificación y drenaje de una matriz extracelular alterada por factores ambientales, nutricionales, inmunológicos y medicamentosos del paciente. Se considera que disminuir la acidez de la matriz extracelular favorece el proceso de reparación tisular. Se conoce bien que por el proceso fisiopatológico de la enfermedad, la artritis reumatoide se caracteriza por tener un ambiente acidificado, principalmente dado por la alteración del metabolismo de la glucosa el cual está desplazada hacia la glucólisis, lo que baja la eficiencia de generación de energía y favorece la producción de lactato deshidrogenasa, el cual se acumula en la matriz y la acidifica, generando un inapropiado funcionamiento de las células inmunitarias y perpetúa el estado inflamatorio. (24). El Lymphomyosot, el Berberis Homaccord, y el Ubichinon compositum por sus efectos antioxidantes participan en la actividad de catalizadores enzimáticos a nivel hepático. Es bien sabido que la artritis reumatoide se caracteriza por un estado hipóxico crónico dado por la angiogénesis desregulada y la disfunción mitocondrial, y en consecuencia se produce una crisis energética lo que perpetúa el estrés oxidativo y mayor daño tisular.

Traumeel, Zeel T, Berberis Homaccord, Discus compositum y Lymphomyosot tienen efectos a nivel inmunológico dados por la modulación de citoquinas proinflamatorias y de la vía de la ciclooxigenasa, lo que permite una mejor respuesta al manejo ya que favorece y refuerza la inmunomodulación.

El Gelsemium Homaccord y Discus Compositum usado en el paciente para la aplicación de biopuntura a nivel de columna cervicodorsal y mano, produjo los efectos buscados en cuanto al manejo del dolor en columna y miembro superior, además basados en los principios de la Medicina Tradicional China, se busca el control de la rigidez, dolor y contractura, trastornos de la columna cervical, calma el SHEN, nutre huesos y articulaciones, nutre y armoniza la sangre (y el yin) (25). El Gelsemium Homaccord refuerza efectos encontrados en otros medicamentos dados por sus propiedades antiinflamatorias y analgésicas, encontrando también un efecto adicional a nivel ansiolítico, y basado en las características del paciente, también se debe abordar.

Es de interés mencionar que, dada la evolución clínica satisfactoria, tendiente a la disminución del dolor y de la limitación funcional, llevó al paciente a reducir los requerimientos de medicamentos alopáticos como AINES y se observó un ahorro ante el uso de glucocorticoides, puesto que el 
paciente además de usar la prednisolona como manejo de base, necesitaba dosis adicionales de diclofenaco y dexametasona, que usaba de manera frecuente para modular transitoriamente su sintomatología. Lo anterior representa una ganancia para el paciente, ya que se sabe bien del riesgo de osteoporosis y deficiencia de vitamina $\mathrm{D}$ asociado al uso periódico de glucocorticoides; también se debe tener en cuenta los efectos adversos de los AINES y en particular para este paciente con riesgo cardiovascular, lo que implicaría posibles compromisos a nivel gastrointestinal, hepático y renal a largo plazo. Otro punto importante a destacar es que, al lograrse un ahorro en el uso de glucocorticoides, se logra una mejor respuesta en los medicamentos biorreguladores usados con el fin de disminuir la respuesta inflamatoria y promover la formación de fibras colágenas, ya que el exceso de glucocorticoides altera la fibroplasia, interfiere en la resolución de la inflamación y pronuncia la respuesta crónica al estrés.

Es importante tener en cuenta que una de las principales barreras en lograr una evolución más favorable es el componente nutricional del paciente. Las elecciones dietarías que fomentan la inflamación y alteran el microbiota intestinal, pueden generar y mantener una desadaptada respuesta inmune. (26)

Teniendo en cuenta los antecedentes del paciente y el momento temporal donde la enfermedad se manifestó, se debe considerar que la respuesta al estrés quirúrgico podría ser un detonante inmunológico, inflamatorio y metabólico. Los procedimientos quirúrgicos producen masivamente citoquinas como IL-1, IL-2, IL-6 e IL-8, las cuales promueven la progresión y amplificación de la inflamación, se activan macrófagos, plaquetas, mastocitos y hay activación del sistema humoral, lo que produce generación de radicales libres de oxígeno y nitrógeno. Estos procesos podrían sobrepasar la capacidad de autorregulación y reventar un sistema ya enfermo (27).

El uso de estos medicamentos multiobjetivo y multicomponente en nuestro paciente potencia el efecto sinérgico de cada uno, llevándonos a una mejor respuesta clínica, potenciando la resolución de la inflamación disminuyendo o autolimitando el progreso de las afecciones articulares causadas por la enfermedad y podría disminuir las manifestaciones extraarticulares de esta por su efecto inmunomodulador.

En la imagen 6. se anexa consentimiento informado del paciente.

\section{Acknowledgements}

Agradecimiento al Dr. Baltazar Guerrero por la orientación durante el desarrollo del presente caso clínico.

\section{$\underline{\text { References }}$}

1. Ankoor S, St. Clair EW. Artritis Reumatoide. Harrison principios de medicina interna. 19a edición; México: McGraw Hill; 2016. 2136-2149) 
2. Gómez A. Nuevos criterios de clasificación de artritis reumatoide. Rev, Reumatología clínica, 2011 Vol. 6. Núm. S3.páginas 33-37.

3. Fernandez-Avila DG, et al. Prevalencia de la artritis reumatoide en Colombia según información del sistema integral de información de la protección social. Rev colomb reumatol. 2019; 26 (2): 83-87.

4. Informe del año 2020 con corte a septiembre del 2019, en la cuenta de enfermedades de alto costo: https://cuentadealtocosto.org/site/artritis-reumatoi

5. M. T. Vicente Herrero, S. Delgado Bueno, F. Bandrés Moyá, M. V. Ramírez Iñiguez de la Torre y L. Capdevila García. Valoración del dolor. Revisión comparativa de escalas y cuestionarios. Rev Soc Esp Dolor. 2018; 25(4): 228-236.

6. M. Herdman, El EuroQol-5D: Una alternativa sencilla para la medición de la calidad de vida relacionada con la salud en atención primaria. Rev ELSEVIER 2001 Vol. 28. Núm. 6.páginas 425-430.

7. Virginia Villaverde García y Alejandro Balsa Criado. Factores pronósticos de la artritis reumatoide. Rev Esp Reumatol 2002;29(1):10-5 .

8. Schneider C. Traumeel - an emerging option to nonsteroidal anti-inflammatory drugs in the management of acute musculoskeletal injuries. Int J Gen Med. 2011;4:225-234.

9. Fearon U, Canavan M, Biniecka M, Veale DJ. Hypoxia, mitochondrial dysfunction and synovial invasiveness in rheumatoid arthritis. Nat Rev Rheumatol. 2016 Jul;12(7):385-97.

10. Educación médica continuada - Heel, Vademecum Medicina Biorreguladora [Internet]. EDUKA -HEEL. 2020 [citado 11 mayo 2021]. Disponible en: https://www.edukaheel.com/es/vademecum

11. FA V. Discus 2020. Educación médica continuada - heel [Internet]. eduka - heel. 2020 [citado 11 mayo 2021]. Disponible en: https://www.eduka-heel.com/es/

12. R.Gottwald,M weiser. Treatment of Rheumatic Disease with a Homeopathic preparation. Biomedical Therapy/Vol/XVIII/No 2.2000 pag 200-216.

13. Birnesser H, Stolt $\mathrm{P}$. The homeopathic antiarthitic preparation Zeel comp. N: a review of molecular and clinical data. Explore (NY). 2007 Jan-Feb;3(1):16-22.

14. Jin GL, Su YP, Liu M, Xu Y, Yang J, Liao KJ, Yu CX. Medicinal plants of the genus Gelsemium (Gelsemiaceae, Gentianales)--a review of their phytochemistry, pharmacology, toxicology and traditional use. J Ethnopharmacol. 2014 Feb 27;152(1):33-52.

15. Yuan Z, Liang Z, Yi J, Chen X, Li R, Wu Y, Wu J, Sun Z. Protective Effect of Koumine, an Alkaloid from Gelsemium Sempervirens, on Injury Induced by $\mathrm{H}_{2} \mathrm{O}_{2}$ in IPEC-J2 Cells. Int J Mol Sci. 2019 Feb 11;20(3):754.

16. Keim, A. P., Slis, J. R., Mendez, U., Stroup, E. M., Burmeister, Y., Tsolaki, N., Gailing, O., \& Goldman, J. (2013). The multicomponent medication lymphomyosot improves the outcome of experimental lymphedema. Lymphatic research and biology, 11(2), 81-92.

17. Alonso Jorge. Fumaria. Tratado de fitofármacos y nutracéuticos. $1^{\circ}$ edición. Argentina. Corpus Editorial y distribuidora. 2007. 470-472.

18. Gründemann C, Garcia-Käufer M, Sauer B, Stangenberg E, Könczöl M, Merfort I, Zehl M, Huber R. Traditionally used Veronica officinalis inhibits proinflammatory mediators via the NF- $\mathrm{BB}$ signalling pathway in a human lung cell line. J Ethnopharmacol. 2013 Jan 9;145(1):118-26. 
19. Klimek-Szczykutowicz M, Szopa A, Ekiert H. Chemical composition, traditional and professional use in medicine, application in environmental protection, position in food and cosmetics industries, and biotechnological studies of Nasturtium officinale (watercress) - a review. Fitoterapia. 2018 Sep;129:283-292.

20. Hua S, Zhang Y, Liu J, Dong L, Huang J, Lin D, Fu X. Ethnomedicine, Phytochemistry and Pharmacology of Smilax glabra: An Important Traditional Chinese Medicine. Am J Chin Med. 2018;46(2):261-297. doi: 10.1142/S0192415X18500143. Epub 2018 Feb 12. PMID: 29433390..

21. Kalmarzi RN, Naleini SN, Ashtary-Larky D, Peluso I, Jouybari L, Rafi A, Ghorat F, Heidari N, Sharifian F, Mardaneh J, Aiello P, Helbi S, Kooti W. Anti-Inflammatory and Immunomodulatory Effects of Barberry (Berberis vulgaris) and Its Main Compounds. Oxid Med Cell Longev. 2019 Nov 19;2019.

22. Rad SZK, Rameshrad M, Hosseinzadeh H. Toxicology effects of Berberis vulgaris (barberry) and its active constituent, berberine: a review. Iran J Basic Med Sci. 2017 May;20(5):516-529.

23. McInnes IB, Schett G. The pathogenesis of rheumatoid arthritis. N Engl J Med. 2011 Dec 8;365(23):2205-19.

24. Qiu J, Wu B, Goodman SB, Berry GJ, Goronzy JJ, Weyand CM. Metabolic Control of Autoimmunity and Tissue Inflammation in Rheumatoid Arthritis. Front Immunol. 2021 Apr $2 ; 12: 652771$.

25. Fundamentos de Acupuntura y Moxibustion de China ,1984.

26. Myles IA. Fast food fever: reviewing the impacts of the Western diet on immunity. Nutr J. 2014 Jun 17;13:61.

27. Senoner, T., Schindler, S., Stättner, S., Öfner, D., Troppmair, J., \& Primavesi, F. (2019). Associations of Oxidative Stress and Postoperative Outcome in Liver Surgery with an Outlook to Future Potential Therapeutic Options. Oxidative Medicine and Cellular Longevity, 2019.

\section{Attachments}

Cabla 2: Criterios de clasificación de Ia EULAR/ACR de artritis reumatoide 2O1O(s)

Los nuevos criterios de AR sólo se aplicarán sobre una determinada población diana que debe tener las

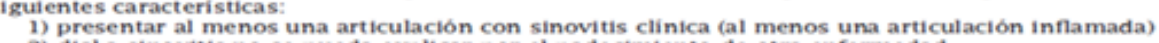
2) dicha sinovitis no se pueda explicar por el padecimiento de otra enfermedad

Un paciente será clasificado de AR si la suma total de las siguientes variables
es $\geq 6$ : A. Afectación articular".

1 articulación articular".
grande afectadas

2-10 articulaciones grandes afectadas

-10 articulaciones pequenas afectadas (con o sin afectación de grandes articulaciones)

$>10$ articulaciones (al menos una pequena)

B. Serologia (al menos se necesita un resultado)

FR Y/o anti-PCC positivos bajos ( 3 valor normal)

FR y/o anti-PCC positivos altos ( $>3$ valor normal)

C. Reactantes de fase aguda

VSG y PCR normales

VSG Y/O PCR elevadas
D. Duración de los sintomas

$<6$ semanas
$<6$ semanas

R: factor reumatoide; anti-PCC: anticuerpos anti-peptido ciclico citrulinado.

- La afectación articular se refiere a cualquier articulación inflamada o dolorosa a la exploración y en la que pueda evi. denciarse mediante pruebas de imagen la sinovitis.

"Articulaciones grandes" se refiere a hombros, codos, caderas, rodillas y tobillos.

positivo a titulos bajos. 


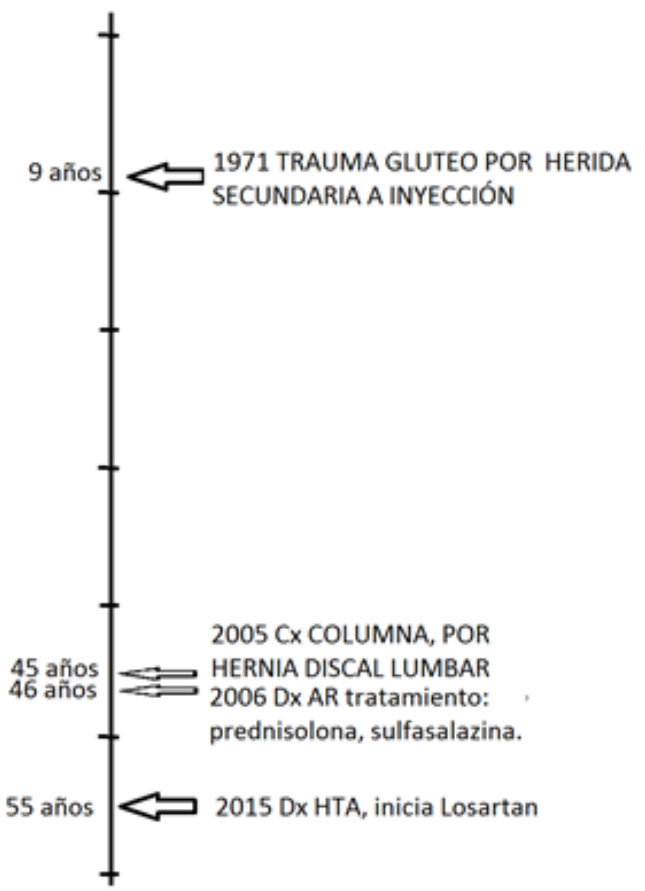

\section{imagen 2}

\section{Paciente CAso clinico $\quad 09 / 01 / 2021$}

CUESTIONARIO DE SALUD EUROCOL-50

Marcue con una cra la mopuesta de cada apartado que mejpr describa su estado de salud en el da de HOY,

\section{Movilidad}

- No tengo problemas para caninar

- Tengo alounos probiemas para camina

- Tengo que estar en la cama

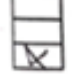

Culdado personal

- No tengo problemas con el cuidado personal

- Tengo algunos pretlemas para lavarme o vestirme

- Soy incapaz de lavarme o vestirmo

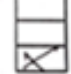

Actividades cotidlanas (p. ef, trabaja, estudac, hacer las tareas

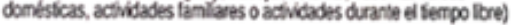

- No vengo problemas para realizar mis actividedes cosilanas

- Tengo algunos problemas para realizar mis actividades cotclanas

- Soy incapaz de realizar mis activides cosflanas

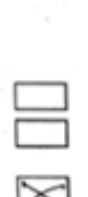

Dolorimalestar

- No tengo dolor ni malestar

- Tengo moderado dolor o malestar

- Tengo mucho dolor o malestar

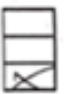

Ansiedad depresion

- No estoy ansioso ni deprimido

- Estoy moderadamente ansioso o deprimido

- Estoy muy ansiosso o deprimido

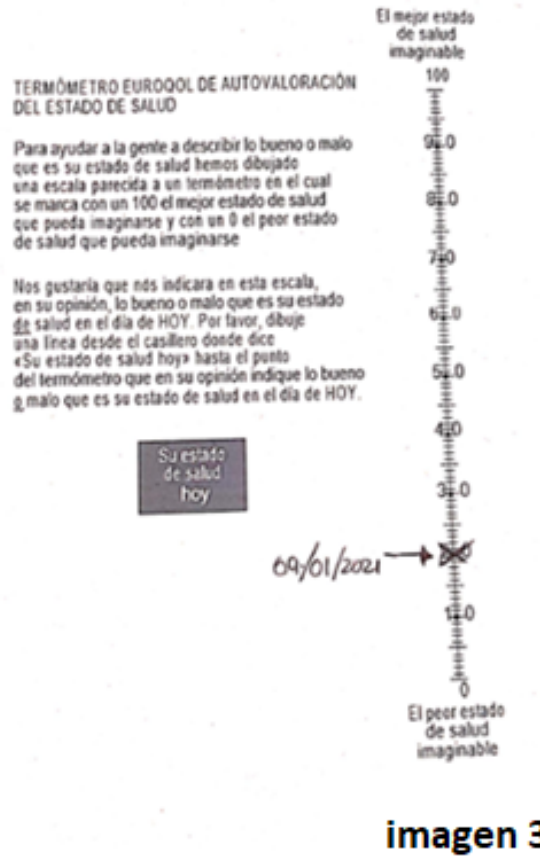




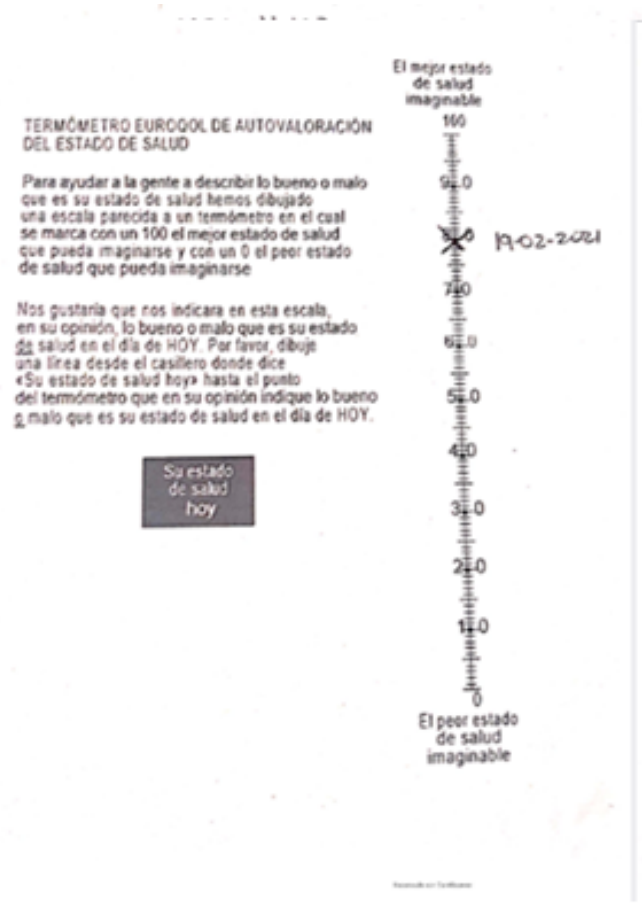

$19 / 02 / 2021$

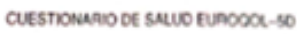

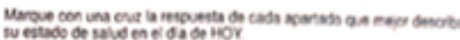

Movatas

No levpo probiemus para canina

Tengo algnos probienus para caring

- Tengo que estar en la cama

Culdado personat

- No wengo probiemas con el cuidads persony

Tengp aigunes protienas para lavame o witime

- Soy incapaz de tavame ovestrme

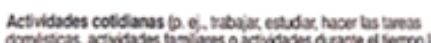

- No enpo problemas para realizar mis activades cetidanas

- Tenge a gunos problemas para rediza mis actividaces cetodanas

- Soy incapar de resilar mis adtidades cosdanas

Dolorimalestar

- No vengo dolor si malestar

- Tengo moderado dotar o malesta.

- Tengo mucho dolor o malistra

Ansiedad depession

- No estoy ansioso ni deprimido

- Estoy moderadamente ansiono o deprimida

- Estoy muy andioso o depinido

\section{imagen 4}

\begin{tabular}{|c|c|c|}
\hline CITA & MEDICAMENTO/PRESENTACIÓN & AREA/VIA DE ADMINISTRACION \\
\hline $1(09 / 01 / 2021)$ & UBICHINON COMPOSITUM (AMPOLLA) & \multirow{3}{*}{ V11, V15, V17, V23, V52, PUNTOS DE DOLOR EN COLUMNA } \\
\hline 1 & DISCUS COMPOSITUM (AMPOLLA) & \\
\hline 1 & TRAUMEEL (AMPOLLA) & \\
\hline 1 & LYMPHOMYOSOT (GOTAS) & 30 gotas en botella de $1000 \mathrm{cc}$ por dia \\
\hline 1 & BERBEEL HOMACCORD (GOTAS) & 30 gotas en botella de $1000 \mathrm{cc}$ por dia \\
\hline 1 & NUXEEL HOMACCORD (GOTAS) & 30 gotas en botella de $1000 \mathrm{cc}$ por dia \\
\hline 1 & ZEEL T (TABLETA) & 1 tabsl, 3 veces al dia. \\
\hline 1 & TRAUMEEL (TABLETA) & 1 tab sl, 3 veces al dia. \\
\hline \multirow[t]{3}{*}{$2(22 / 01 / 2021)$} & GELSEMIUM (AMPOLLA) & V11, V15 \\
\hline & TRAUMEEL + DISCUS COMPOSITUM & V20, V23, v52 \\
\hline & ZEELL T + TRAUMEEL & E36, E34 \\
\hline \multirow[t]{3}{*}{$3(27 / 01 / 2021)$} & TRAUMEEL + DISCUS COMPOSITUM & $\mathrm{V} 23, \mathrm{~V} 52$ \\
\hline & GELSEMIUM (AMPOLLA) & V11, ID3 \\
\hline & TRAUMEEL & Rodillas $36 \mathrm{E}$ \\
\hline \multirow[t]{2}{*}{$4(29 / 01 / 2021)$} & ZEEL + TRAUMEEL (AMPOLLA) & $E 34, E 36, V 23, V 52$ \\
\hline & GELSEMIUM (AMPOLLA) & V11, V17 \\
\hline \multirow[t]{2}{*}{$5(5 / 2 / 2021)$} & ZEEL T + DISCUS COMPOSITUM +TRAUMEEL & V11, v15, v17, v23, v20 \\
\hline & ACUPUNTURA & P7, R6, B6, E36, B10 \\
\hline \multirow[t]{2}{*}{$6(19 / 02 / 2021)$} & GELSENIUM & V11, V15, V17, ID3 \\
\hline & ZEELL T+ TRAUMEEL & V23, V2O, B6, E36, E34 \\
\hline \multirow[t]{2}{*}{$7(14 / 05 / 2021)$} & GELSENIUM (AMPOLLA) & $\mathrm{V} 11, \mathrm{~V} 15, \mathrm{~V} 17, \mathrm{ID} 3$ \\
\hline & ZEEL T + TRAUMEEL (AMPOLLA) & $\mathrm{V} 23, \mathrm{~V} 20, \mathrm{~B} 6, \mathrm{E} 36, \mathrm{~B} 10$ \\
\hline
\end{tabular}


NOMBRE DEL PACIENTECAIIOS nrtuto Raviríz

NUMERO IDENTIICACION Cedula 116.357566

1) Declaro que me han explicado y he comprendido satisfactoriamente la naturaleza y propósitos de las forapias y procedimientos de medicina altomativa y complementaria propuestos y realizados por la médica tratante, y que son ef complementar los tratamientos de la o las entermedades por las cualos consulto mediante procedimientos y terapias diferentes a las de la medicina convoncional. He sido informado que la medicina alternativa no tiene su énfasis on ol diagnóstico do la enfermedad, por lo cual para el adecuado diagnóstico de mi caso, debo do la entermersos acudir a los diferentes procedimientos, exámenes e interconsultas con los diversos debo suspender los tratamientos que me han sido prescritos por parte de ollos. Igualmente me ha sido aclarado que si bien el médico tratante ostá facultado para gromular medicamentos convencionales, preferiblemento debo acudir a consulta con los médicos de la medicina convencional para que estos me sean formulados. También me han sido aclarado todas las dudas así como las otras alternativas de tratamiento que existen. Además se me ha informado que no existen garantias absolutas del resultado de las terapias y procedimientos realizados, y que la duración del tratamiento y su pronóstico dependen del mismo caso a tratar.

2) He sido informado que como todo procedimiento médico, las terapias y tratamientos de medicina alternativa pueden tener posibles riesgos y complicaciones todos los cuales me han sido explicados, haciéndome énfasis en que los principales riesgos y complicaciones de las terapias y tratamientos que pueden presentarse son: A) Reacciones alérgicas que pueden ser de leves a severas debidas a las aplicaciones de las terapias ordenadas y realizadas especialmente a los anestésicos locales utilizados en la Terapia Neural y de sueros endovenosos; a los medicamentos homeopáticos, a los nutrientes y medicamentos tanto naturales como farmacológicos formulados, y a cualquier otro procedimiento o terapia de medicina alternativa realizado. B) Infecciones y lesiones por terapias invasivas con agujas utilizadas en la aplicación de sueros, Acupuntura y la Terapia Neural C) Exacerbación de sintomas recientes o antiguos.

3) Por tratarse de tratamientos de medicina alternativa y complementaria he sido informado que debo seguir todas las instrucciones y recomendaciones dadas, y que en caso de presentarse agravamiento o persistencia del cuadro clínico por el cual consulto, debo informar al médico tratante y en caso de no lograr comunicación con este por problemas técnicos que impidan dicha comunicación por teléfono, medios digitales etc. o si el caso se torna grave, debo asistir al servicio médico al cual pertenezco, o en su defecto a un facultativo idóneo de la

imagen 6 